\title{
COMPUTERIZED MORPHOMETRY OF EPITHELIAL FIMBRIAE COMBINED WITH ARTIFICIAL INTELLIGENCE IN BRCA CARRIERS MAY IDENTIFY PATIENTS AT RISK FOR DEVELOPING OVARIAN CANCER; A PRELIMINARY STUDY
}

\author{
A. Amit, E. Sabo, A. Petruseva, A. Reiss, G. Klorin \\ Rambam Health Care Campus, Haifa, Israel
}

Objectives: Serous ovarian tumors may originate in epithelial cells of the fallopian tubes. Computerized morphometry was able to find significant alterations in the fallopian tube epithelium of healthy BRCA carriers. The purpose of this study was to identify a subgroup of BRCA carriers that may be at risk of developing serous ovarian cancer by evaluation of the epithelial nuclear symmetry in the fallopian tubes.

Methods: Four groups of patients were analyzed; healthy patients, ovarian cancer patients , BRCA carriers, and BRCA non-carriers. All fallopian tubes appeared normal by H\&E examination. The ImageProPlus software was used to assess the nuclear symmetry of 65 fimbriae epithelium cells and an artificial neural network algorithm aided in detecting a subpopulation among fimbriae of healthy BRCA carriers at risk for ovarian cancer .

Results: Significant differences were found between healthy patients and ovarian cancer patients, and between BRCA carriers and non-carriers. The algorithm was able to accurately predict BRCA carriers with associated ovarian cancer based on fallopian tube nuclear symmetry characteristics.
Conclusions: These results reinforce the hypothesis that fimbriae epithelium cells of BRCA carriers' may undergo early-stage changes that may predict progression toward malignancy. Artificial intelligence may identify patients at high risk for malignancy initiated in the fallopian tubes.

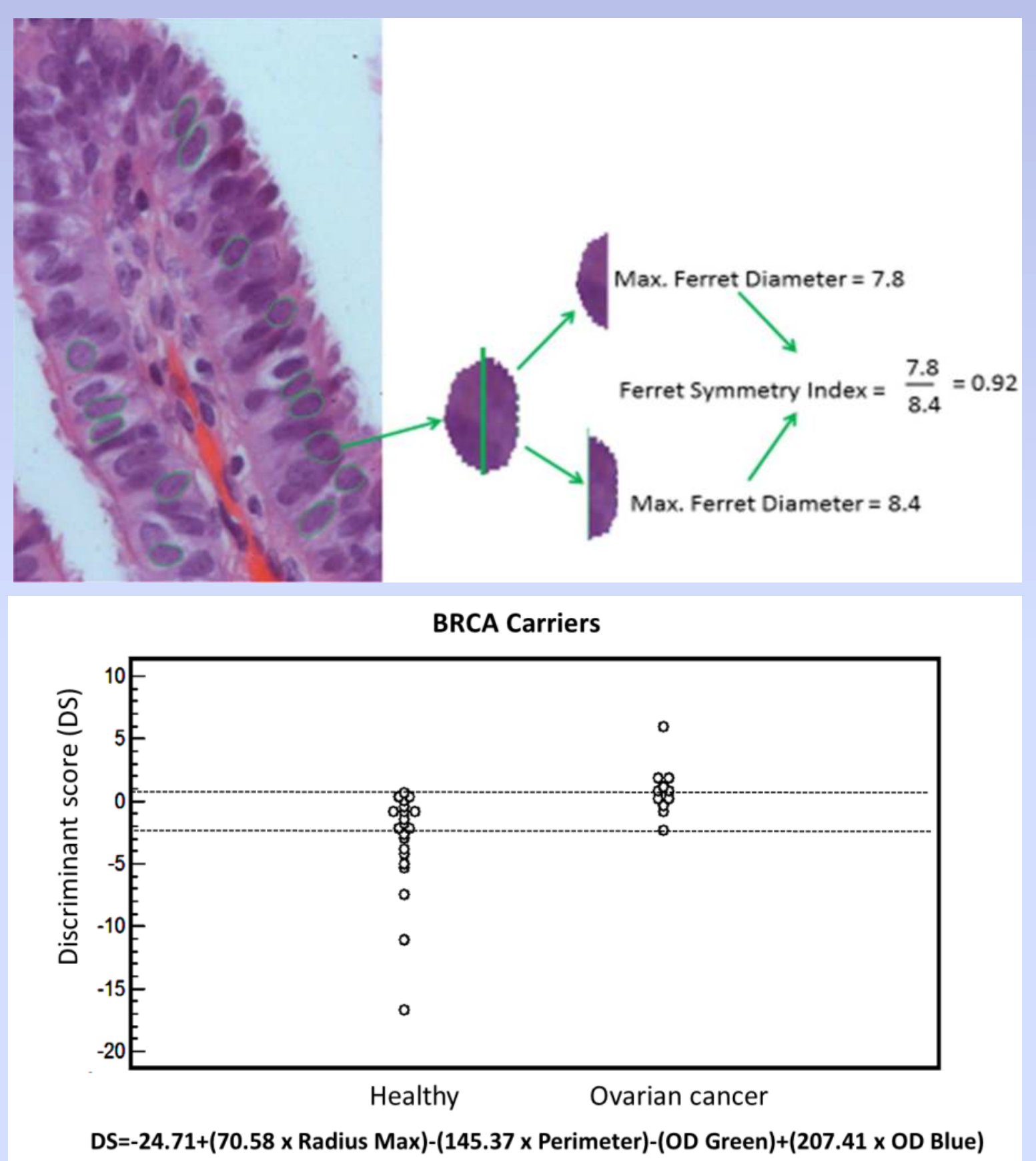

\section{Artificial Neural Network models}

\section{Comparison of the coefficients symmetry in the fallopian tubes nuclei between patients with and without ovarian cancer}

\section{All BRCA carriers and not carriers}

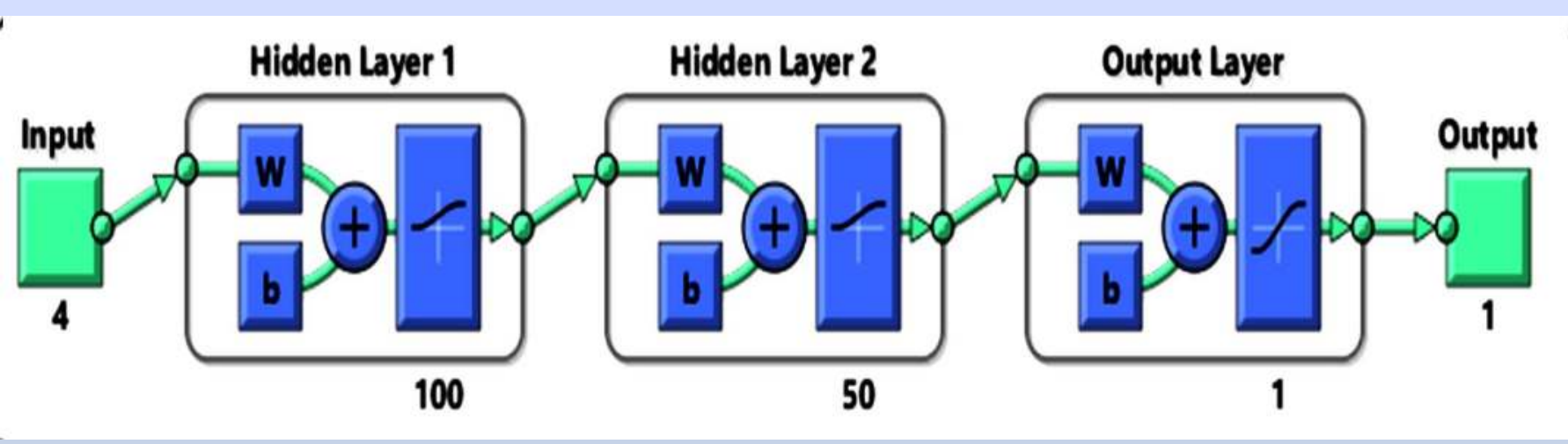

Sensitivity: $90.3 \%$, Specificity: $94.1 \%$, General accuracy: 92.3.
Within the BRCA population only

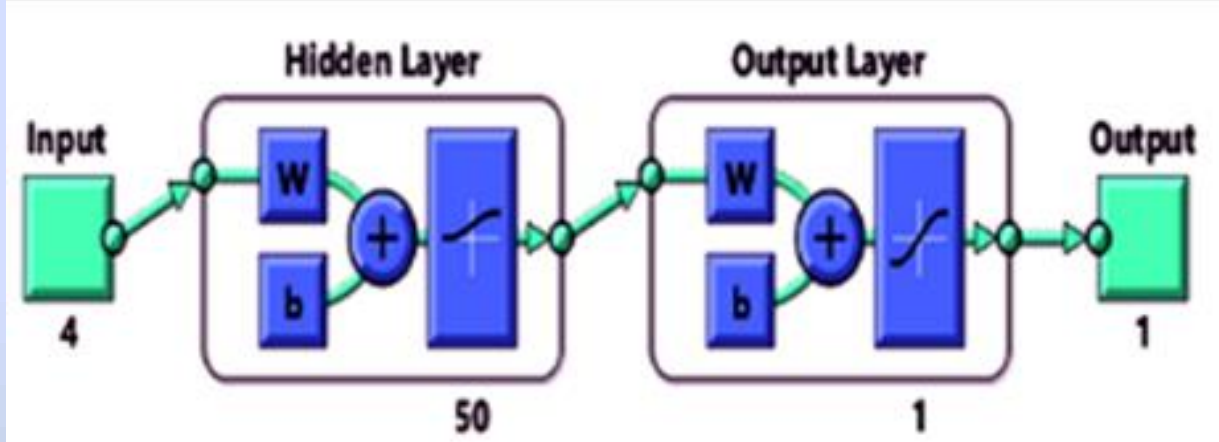

Sensitivity: $90.9 \%$, Specificity: $95.2 \%$, General accuracy: 93.8. 